\title{
JEAN CASSINET
}

\section{La réédition de l'œuvre majeure de Stieltjes}

Annales de la faculté des sciences de Toulouse $6^{e}$ série, tome $4, \mathrm{n}^{\mathrm{o}} 1$ (1995), p. JI-JIV

<http://www.numdam.org/item?id=AFST_1995_6_4_1_JR1_0>

(C) Université Paul Sabatier, 1995, tous droits réservés.

L'accès aux archives de la revue «Annales de la faculté des sciences de Toulouse » (http://picard.ups-tlse.fr/ annales/) implique l'accord avec les conditions générales d'utilisation (http://www.numdam.org/conditions). Toute utilisation commerciale ou impression systématique est constitutive d'une infraction pénale. Toute copie ou impression de ce fichier doit contenir la présente mention de copyright.

\section{Numdam}

Article numérisé dans le cadre du programme Numérisation de documents anciens mathématiques http://www.numdam.org/ 


\title{
La réédition de l'œuvre majeure de Stieltjes
}

\author{
JeAn CASSinet ${ }^{(*)}$
}

En 1994-1995, la Société Mathématique des Pays-Bas d'une part et l'Université Paul Sabatier de Toulouse ont commémoré le centième anniversaire de la mort de Thomas Jan Stieltjes (1856-1894), ce mathématicien d'origine hollandaise devenu entre 1886 et 1894 une des gloires de la Faculté des Sciences de Toulouse. Après avoir donné l'indiscutable nom de Fermat au premier amphithéâtre de mathématiques dans l'université nouvellement installée dans la périphérie toulousaine, sur proposition du professeur Roger Huron, le nom de Stieltjes fut attribué en 1966 au deuxième amphithéâtre de mathématiques de cette université. Cette décision consacrait donc un mathématicien d'origine hollandaise comme gloire toulousaine digne de partager avec Fermat la postérité universitaire. Cet insigne honneur est mérité non seulement par l'importance des travaux mathématiques de Stieltjes, mais aussi par le rôle essentiel que son passage, pourtant écourté par une mort prématurée, a joué dans le développement des mathématiques en l'Université de Toulouse.

Parmi une œuvre considérable touchant à de nombreux domaines des mathématiques, son œuvre majeure reste sans conteste son mémoire sur les fractions continues ${ }^{(1)}$, où il introduit la notion connue de nos jours sous le nom de "mesure de Stieltjes" autrement dit un élément nouveau dans la théorie de l'intégration.

Cet imposant, important et magnifique mémoire est le point d'orgue et le chant du cygne de ce mathématicien exceptionnel. En effet atteint par une maladie incurable pour l'époque, avec le soutien de son maître et indéfectible ami Charles Hermite, Stieltjes a consacré toute son énergie, jusqu'à son dernier souffle de vie, pour achever son œuvre.

(*) Département de Mathématiques, Université Paul Sabatier, 118 route de Narbonne, F-31062 Toulouse Cedex (France)

(1) Recherches sur les fractions continues, Ann. Fac. Sci. Toulouse 8 (1894) pp. J1J122 et 9 (1895) pp. A1-A47 
On ne peut à ce propos faire mieux que citer cet extrait de la très belle communication que fit, le 2 décembre 1973, le professeur Roger Huron à l'Académie des Sciences et Belles-Lettres de Toulouse où, parlant des dernières années de la vie de Stieltjes, il $\operatorname{dit}^{(2)}$ :

"Sur le plan physique c'est une suite d'échecs, mais sur le plan de la volonté de réalisation d'un dessein que, me semble-t-il, on doit considérer comme l'élaboration d'une œuvre d'art, c'est une victoire."

Le 20 octobre 1892, Stieltjes envoyait à Hermite une note contenant un théorème et sa démonstration concernant les fractions continues. Cette note sera publiée dans les mémoires de l'Académie des Sciences de Paris. Hermite lui répondra immédiatement pour lui manifester son admiration :

"Vous êtes un merveilleux géomètre, les recherches nouvelles sur les fractions continues algébriques que vous me communiquez sont un modèle d'invention et d'élégance; ni Gauss, ni Jacobi ne m'ont jamais causé plus de plaisir et je vous envoie mes félicitations en vous demandant si je dois publier dans les Compte Rendus la partie mathématique de votre lettre."

En juin 1893, après une interruption due à son état de santé, Stieltjes travaille encore à son mémoire sur les fractions continues "qui avance doucement" écrit-il à Hermite.

Après un nouveau temps de repos et de soins, il relance sa recherche sur les fractions continues en octobre 1893. Coup sur coup, deux lettres parviennent à Hermite sur le sujet qui sera désormais le centre de ses préoccupations. Aussi le 5 octobre, Hermite lui écrit :

"Votre travail sur les fractions continues sera l'un des plus considérables et des plus beaux que vous ayez produits. C'est un vaste champ entièrement nouveau que vous avez ouvert à l'Algèbre et à l'Analyse..."

Malgré de nombreuses difficultés de santé et un séjour de repos en Algérie, au prix d'efforts redoublés et surhumains, le 25 mai 1894, il peut enfin écrire :

" J'ai fait remettre à monsieur Berson le manuscrit de mes recherches sur les fractions continues. Je vais maintenant m'octroyer un peu de repos."

Dès le 29 mai, Hermite manifeste son enthousiasme pour l'œuvre de son ami :

"Votre mémoire sur les fractions continues est une œuvre analytique d'une importance capitale et j'apprends avec la plus grande satisfaction

(2) Roger HURon, Le destin hors série de Thomas-Jan Stieltjes, Mémoires de l'Académie des Sciences, Inscriptions et Belles-Lettres de Toulouse. Vol. 136, $15^{\text {ème }}$ série, V (1974), pp. 93-125 
qu'elle va s'imprimer et être prochainement publiée. Comme d'autres de vos productions, elle sera un stimulant pour mes recherches et je me joindrai à ceux qui s'engagent après vous dans la voie féconde que vous avez ouverte."

$\mathrm{Au}$ cours de l'été, l'impression du mémoire avance et Hermite lui en demande un résumé pour l'Académie. À l'Académie, le rapport sur le mémoire de Stieltjes est fait par Poincaré et il est, on ne peut plus, élogieux... Sa conclusion mérite la citation :

"Le travail de Monsieur Stieltjes est donc un des plus remarquables mémoires d'Analyse qui ait été écrit dans ses dernières années; il s'ajoute à beaucoup d'autres qui ont placé leur auteur à un rang éminent dans la science de notre époque. La commission a l'honneur de proposer à l'Académie d'accorder à $M$. Stieltjes le plus haut témoignage de son approbation en ordonnant l'insertion de son mémoire "sur les fractions continues" dans le Recueil des Savants Etrangers et elle émet le vœu qu'un prix puisse lui être accordé sur la fondation Lecomte."

Bien plus tard et de nos jours, c'est Jean Dieudonné qui notera l'importance du mémoire de 1894 en soulignant ses qualités esthétiques :

" Ce dernier (Stieltjes) y posait et résolvait, avec une rare élégance, tout une série de problèmes d'un type nouveau sur les fonctions analytiques et les fonctions d'une variable réelle $e^{(3)}$."

Cependant lorsque paraît la première partie du mémoire la fin est proche. Le 15 décembre une dernière lettre d'Hermite à Stieltjes, où il lui souhaite que les forces lui reviennent, restera sans réponse. En effet, Stieltjes mourra le 31 décembre 1894. Il avait 38 ans depuis deux jours.

Le mémoire sur les fractions continues, à cause de sa longueur, n'a pu être entièrement publié dans le volume des Annales de Toulouse de 1894, avant la mort de Stieltjes. Aux 122 premières pages de 1894, succéderont les 47 dernières publiées en 1895 par les soins de son collègue et ami Eugène Cosserat lequel, après l'avoir remplacé durant sa maladie, aura ensuite le redoutable honneur de lui succéder dans la chaire toulousaine de Calcul Différentiel et Intégral.

Le volume de 1895 des Annales de la Faculté des Sciences de Toulouse commence par un hommage collectif du Comité de Rédaction dont il était par ailleurs un des membres les plus actifs.

(3) Abrégé d'Histoire des Mathématiques (Herman, 1978, Tome 2, chap. XI. Intégration et mesure par Jean Dieudonné (pp. 272-274; III-mesures de Stieltjes et de Radon) 
“ Le 31 Décembre 1894 est mort à 38 ans, après une longue maladie, Thomas Jean Stieltjes, professeur du Calcul Différentiel et Intégral à la Faculté des Sciences de Toulouse. Un des membres du Comité des Annales, M. E. Cosserat, a bien voulu analyser 82 notes ou mémoires publiés par Stieltjes dans divers recueils et en a fait précéder l'analyse par une notice biographique. Le comité a pensé que la publication de cette analyse était le meilleur hommage qu'il pût rendre immédiatement à la mémoire de l'un des hommes dont le caractère et le talent ont fait le plus d'honneur à la Faculté..."

La notice de Cosserat est considérable et occupe 64 pages de ce numéro des Annales qui suit la mort de Stieltjes. L'hommage est à la hauteur du grand mathématicien qui vient de s'éteindre. En outre, la publication de la fin de l'œuvre sur les fractions continues parachevait cet hommage des universitaires toulousains.

On comprendra qu'en ce centième anniversaire de la mort de Stieltjes, les Annales de la Faculté des Sciences de Toulouse aient jugé bon de rééditer ce chef d'œuvre mathématique encore très actuel que constitue le mémoire "Recherches sur les fractions continues" et la note qui le prépare. Étant donné l'importance du texte, celui-ci sera publié au cours de quatre numéros de notre revue, revue à laquelle, rappelons-le, Stieltjes avait consacré jadis une part importante de son temps en vue de lui donner un grand éclat dans le monde mathématique de son époque. 